\title{
The acquisition of Multi-drug resistant bacteria in patients admitted to COVID-19 intensive care units: a monocentric retrospective case control study
}

\section{Elisa Gouvêa Bogossian ( $\nabla$ elisagobog@gmail.com )}

Erasmus Hospital - université Libre de Bruxelles https://orcid.org/0000-0002-2062-465X

\section{Fabio Silvio Taccone}

Erasmus Hospital, Intensive Care department , Université Libre de Bruxelles

Antonio Izzi

Erasmus Hospital, Intensive Care department, Université Libre de Bruxelles

Nicolas Yin

Erasmus Hospital, Microbiology Department, Université Libre de Bruxelles

\section{Alessandra Garufi}

Erasmus Hospital Intensive Care Department, Université Libre de Bruxelles

\section{Stephane Hublet}

Erasmus Hospital Department of Anaesthesiology and Intensive Care Medicine, Université Libre de Bruxelles

\section{Hassane Nijmi}

Erasmus Hospital, Intensive Care Department, Université Libre de Bruxelles Julie Gohram

Erasmus hospital, Intensive Care Department, Université Libre de Bruxelles

\section{Baudoin Byl}

Erasmus Hospital, Epidemiology and Infection Control Department, Université Libre de Bruxelles

\section{Alexandre Brasseur}

Erasmus Hospital , Intensive Care Department, Université Libre de Bruxelles

\section{Maya Hites}

Erasmus Hospital, Infectious and Tropical Diseases Department, Université Libre de Bruxelles

\section{Jean-Louis Vincent}

Erasmus Hospital, Intensive Care Department, Université Libre de Bruxelles

\section{Jacques Creteur}

Erasmus Hospital, Intensive Care Department, Université Libre de Bruxelles

\section{David Grimaldi}

Erasmus Hospital, Intensive Care Department, University Libre de Bruxelles 
Keywords: antimicrobial resistance, Enterobacteriaceae, Subarachnoid hemorrhage, critically ill, infection control

Posted Date: September 8th, 2020

DOI: https://doi.org/10.21203/rs.3.rs-67837/v1

License: (1) This work is licensed under a Creative Commons Attribution 4.0 International License. Read Full License 


\section{Abstract}

Background: Whether the risk of multidrug resistant bacteria (MDRB) acquisition in the intensive care unit (ICU) is increased during the COVID-19 crisis is unknown. Our aim was to measure the rate of MDRB acquisition in patients admitted in COVID-19 ICU and to compare it with pre-COVID-19 controls.

Methods: This single center case control study included adult patients admitted to COVID-19 ICUs for more than 48h. Patients were screen twice a week for MDRB carriage during ICU stay. We compared the rate of MDRB acquisition of COVID-19 patients with a cohort of patients admitted for subarachnoid hemorrhage (SAH) and matched on length of ICU stay using a competing risk analysis.

Results: Among 72 patients admitted to the COVID-19 ICUs, 24 (33\%) patients acquired 31 MDRB during ICU stay. The rate of MDRB acquisition was 30/1000 patient-days. Patients that acquired MDRB had received more antimicrobial therapy [22 (92\%) vs $34(78 \%, p=0.05]$ and had a longer exposure time [12 days (8-18) vs 5 days (2-18), $p=0.02$ ]. The rate of MDRB acquisition in matched SAH patients was $18 / 1000$ patient-days. When compared to SAH retrospective cohort, being admitted to a COVID-19 ICU was associated with a numerically higher proportion of MDRB acquisition. This association did not reach statistical significance in the multivariable competing risk analysis (sHR 1.71 (Cl 95\% 0.93-3.21).

Conclusion: Acquisition of MDRB was frequent during the COVID-19 first wave in ICU patients. Despite physical isolation, it was similar to patients admitted to the same ICU in previous years.

\section{Introduction}

The respiratory infection COVID-19 caused by severe acute respiratory syndrome coronavirus 2 (SARSCoV-2) has spread worldwide, being considered pandemic by the World Health Organization since March 11, 2020. [1]

During this pandemic, health care systems worldwide became overloaded and experienced shortages of intensive care unit (ICU) beds [2] and protective personal equipment (PPE). [3] In the most severely affected countries, ICUs were filled with only COVID-19 patients, and named "COVID-19 ICUs"; new ICUs and hospital beds had to be opened,[4] health care professionals were reassigned and started working longer hours. These factors can contribute to a decreased adherence to infection prevention and control measures and, combined with a high antimicrobial selection pressure,[5] may facilitate the emergence of antimicrobial resistance (AMR). [6]

AMR is a major health problem, [7] responsible for high morbi-mortality [8,9] and an elevated economic burden. [10] The ICU is one of the hospital locations where patients have the highest risk of acquiring multi drug resistant bacteria (MDRB). [11]

However, until now, the risk of MDRB acquisition in the ICU during COVID-19 crisis remains unknown; whether this risk of MDRB acquisition is higher in COVID-19 units compared to normal functioning ICU 
has not been studied.

The aim of this study is to describe the rate of MDRB acquisition in COVID-19 ICUs compared with the MDRB acquisition in the same department before the COVID-19 crisis.

\section{Methods}

\section{Study design and setting}

We conducted a single center retrospective case-control study of COVID-19 patients admitted to the ICUs of Erasme Hospital, Brussels, Belgium from March to April 2020. The ethics committee approved this study (P2020/371) and waived the need for an informed consent.

Under normal circumstances, our ICU operates with 30 beds divided into five ICUs with 6-single rooms each. We perform nasal and rectal surveillance cultures upon ICU admission and twice a week thereafter. Colonization by MRSA, ESBL, CPE and VRE is indicated at the room door and requires health care workers to wear gloves and gown before entry. We have a meeting with the antibiotic stewardship team twice a week.

\section{COVID-19 crisis management}

During COVID-19 crisis, we increased our ICU beds from 30 to 39, by opening 5 beds in the existing ICUs and building another 4-beds ICU in two operating rooms. Only one 6-beds ICU remained available for nonCOVID-19 admissions. The remaining 33 beds were reserved for COVID-19 patients with a high risk of invasive mechanical ventilation. Patients with stable clinical condition on CPAP remained in the wards.

\section{Patients' selection}

\section{Cases:}

All patients admitted to the COVID-19 ICUs of Erasme Hospital between 15/03 and 30/04, regardless of the final etiological diagnosis, were eligible for inclusion; the sole exclusion criterion was an ICU stay < 48h. These patients were considered as cases and are named COVID-19 patients in this manuscript.

\section{Controls:}

The admission to the non-COVID 19 ICU was scarce during the study period. Therefore, it was not possible to use this population as control. Given the unusual duration of mechanical ventilation in COVID19 patients, [12] we compared them to patients obtained from a preexisting subarachnoid hemorrhage (SAH) institutional database cohort. [13] These patients were admitted to our ICU from January 2016 2019. Severe SAH patients are young with few comorbidities and can have prolonged ICU length of stay (LOS) [14] and duration of mechanical ventilation. [15] Patients were matched 1:1 according to ICU LOS and the use of mechanical ventilation. Patients with ICU LOS less than ten days were matched with controls with a difference of \pm 2 days, and patients with ICU LOS > 10 days were matched with controls 
with a $\pm 20 \%$ difference in the LOS. When several controls could match one COVID-19 patient we selected the control with the closest ICU LOS.

\section{Data collection and endpoints:}

Demographic data and severity scores were collected at ICU admission. We defined the length of exposure as the duration between ICU admission and the day of MDRB acquisition, or between ICU admission and ICU discharge in patients without MDRB acquisition. We collected the following data during the exposure time: antimicrobial use, presence of central venous catheter, urinary tract catheter, mechanical ventilation and the occurrence of surgery. The primary endpoint was the rate acquisition of MDRB in the COVID 19 units.

\section{Microbiology data:}

We considered patients MDRB + when a MDRB was found in any microbiological specimen. Patients that were MDRB+ within $48 \mathrm{~h}$ after admission were considered index cases. Patients that acquired MDRB during ICU stay were considered new cases. Patients that didn't acquire MDRB during ICU stay were considered MDRB -. We collected the presence of possible cross-transmission. Cross-transmission was suspected if a patient acquired a MDR pathogen with the same antimicrobial susceptibility and resistance mechanism than another patient hospitalized at the same time in the same unit.

In our center, we perform routine surveillance cultures (rectal swab, tracheal aspirate and urinary cultures) on admission and then twice a week throughout the ICU stay.

For MDRB detection, rectal swabs are streaked onto selective plates as follow: chromID $®$ CARBA SMART agar (bioMérieux, France) for the detection of carbapenemase-producing Enterobacteriaceae; MacConkey agar containing ceftazidime (bioTRADING, Netherlands) for the detection of third generation cephalosporin-resistant Pseudomonas aeruginosa, Klebsiella spp. and Enterobacter spp.; chromID ${ }^{\circledR}$ VRE agar for the detection of Vancomycin-resistant Enterococcus faecium. Identification of MDRB is performed using matrix-assisted-laser desorption ionization-time of flight analysis (MALDI-TOF).

Antimicrobial resistance was defined according to breaking points recommended by the European Committee on Antimicrobial Susceptibility Testing (EUCAST) [16] using VITEK2 and disk diffusion method. Carbapenemases OXA-48, KPC, NDM, VIM and IMP were detected via Polymerase Chain Reaction (PCR) analysis or Coris Resist-5 0.O.K.N.V. antigenic detection (Coris BioConcept, Belgium). VanA and VanB genes were detected via PCR analysis. ESBL-producing Enterobacteriaceae and ampC derepression were identified using detection of synergy on disk diffusion test as recommended by EUCAST. For methicillin-resistant S.aureus detection, nasopharyngeal swabs were streaked onto ChromID ${ }^{\circledR}$ MRSA selective plates. MDR Pseudomonas and Acinetobacter spp. were defined as recommended considering antimicrobial resistance phenotype. [17]

Statistical analysis: 
Categorical variables are reported as count (\%), continuous data that were normally distributed as mean \pm standard deviation (SD) and skewed data as median [interquartile range].

Incidence of MDRB acquisition was calculated by dividing the number of new cases by the total number of patients admitted to the COVID-19 ICUs. The incidence rate was calculated by dividing the number of new cases by the total number of patient-days in the COVID-19 ICUs. We compared MDRB+ and MDRB patients in the COVID 19 cohort using Student's T-test, Mann-Whitney test, $\chi^{2}$ test or Fisher's exact test, as appropriate. In order to identify factors associated with the acquisition of MDRB, we compared patients admitted to COVID 19 ICUs with SAH patients admitted to our ICUs in previous years (2016-2019). Cumulative incidence function of the acquisition of MDRB was used to describe the probability of MDRB acquisition at a given time. The Gray's test was used to test for the differences. Univariate and multivariable regression analyses were performed using the Fine and Gray competing risks proportional hazards regression model. Death was considered as a competing risk factor for the development of MDRB. In the multivariable model only variables that had p-value less than 0.2 by univariate analysis were considered.

All tests were two-tailed and a p value $<0.05$ was considered as statistically significant. Data were analyzed using IBM ${ }^{\circledR}$ SPSS ${ }^{\circledR}$ Statistics software, version 26 for Macintosh (IBM, Armonk, NY).

\section{Results}

\section{Characteristics of patients admitted in COVID-ICUs}

We identified 75 patients admitted to COVID-19 units during the studied period. Three patients were excluded (ICU stay < 48h) letting 72 included patients representing 1104 patient-days of exposure. COVID19 was diagnosed in 69 out of the 72 patients; the remaining 3 non-documented COVID-19 patients were kept in the analysis. Of note, five patients (7\%) were MDRB carriers at ICU-admission.

Fifty-two patients (72\%) were treated with mechanical ventilation. The median ICU LOS in survivors was 33 days (16-52).

\section{Characteristics of MDRB acquisition}

Among the 72 patients, 24 (33\%) acquired 31 MDRB (7 patients acquired more than one MDRB) during their ICU stay. The incidence rate of MDRB acquisition was 30/1000 patient-days. Figure 1 shows the isolated MDRB that were mostly Enterobacteriaceae. No MRSA were isolated. We identified 16/31 (52\%) suspected cross-transmission events involving mainly ESBL K. pneumoniae, AmpC derepressed $E$. aerogenes and Vancomycin resistant $E$. faecium. MDRB positive and negative patients had similar demographic characteristics and severity at admission. Exposure time was longer in MDRB+ patients and antimicrobial therapy was more frequently used. In terms of clinical outcome, ICU and hospital mortality were similar between the two groups. These results are shown in table 1. 
To evaluate if the MDRB acquisition risk had changed during COVID-19 pandemic, we compared the COVID-19 patient cohort with a retrospective cohort of SAH patients hospitalized in the same ICU matched on the need for mechanical ventilation and the ICU LOS. Characteristics of both cohorts are shown in Table 2; SAH patients had a shorter duration of mechanical ventilation and received less antimicrobial therapy during exposure time.

In SAH patients, the proportion of patients with MDRB acquisition was lower ( $21 \mathrm{vs} 32 \%, p=0.2)$ than in COVID-19 patients; MDRB acquisition rate was 18/1000 SAH patient-days compared to the $30 / 1000$ COVID 19 patients-day $(p=0.08)$. Figure 2 shows the cumulative incidence of MDRB acquisition of both cohorts.

In multivariable analysis using a competitive risk model, we observed that being admitted to COVID-19 ICUs was not independently associated with MDRB acquisition [sHR 1.71 (CI95\%: 0.93-3.12), p=0.08] (table 3). The univariate comparison between MDRB+ and MDRB- patients is shown in the ESM table 1.

\section{Discussion}

In this retrospective cohort of patients admitted to COVID-19 ICUs for suspected or confirmed SARS-CoV-2 infection, the rate of MDRB acquisition was 30/1000 patients-days. After adjustment and competitive risk analysis, the risk of MDRB acquisition tended to be higher in COVID-19 ICUs compared to control usual ICU.

The rate of acquisition of MDRB is highly dependent on local parameters, and thus vary greatly across centers and countries. [18] In another Belgium center $29 \%$ of patients acquired MDRB a proportion slightly higher but comparable to our control cohort. [19]

Our study was not designed to explore whether the risk of MDRB acquisition is related to the COVID-19 per se or to the disruption in hospital functioning during the pandemic crisis. In the hospital setting, dissemination of MDRB happens through either cross-transmission or environmental sources, being favored at the individual level by antimicrobial therapy selection pressure [20]. In the ICU environment, cross transmission ranges from $23 \%-53 \%$ of patients' contacts $[21,22]$ due to the frequent and complex cares, which facilitates the contamination of health care workers' hands and, consequently, the dissemination of MDRB [20]. Applying infection prevention and control measures and monitoring their observance are key factors to prevent MDRB spreading. [23]

The possibility of dissemination of MDRB during a viral pandemic had been theoretically mentioned [24], but as many previous pandemic occurred before the antimicrobial resistance era, no data was available before the SARS-CoV-2 pandemic, including the H1N1 pandemic. Experts have raised concern about the dissemination of MDRB during the COVID-19 pandemic $[6,25,26]$ and a report indicates an increase in blood stream infection [27]. In line with these concerns, we observed a high rate of MDRB acquisition in COVID-19 patients at least as high as in non-COVID-19 patients. The rate of MDRB acquisition in the COVID-19 ICUs was numerically higher, although not statistically different, than the rate in a control 
cohort of patients hospitalized in the previous years in the same ICUs. The lack of statistical significance may be due to the limited power of our study. Moreover, one can consider that 2 factors may have limited the MDRB acquisition rate: 1) COVID-19 patients were admitted to ICUs that had been entirely emptied and cleaned in contrast with normal ICU admission, which occurs in units where MDRB carriers are already present and 2) the physical isolation of COVID-19 patients should have provided an efficient barrier to MDRB cross-transmission.

The high rate of MDRB acquisition may be related to several difficulties we experienced during this pandemic. We faced a gown shortage and needed to use the same gown for several patients. Considering the difficulty of undressing PPE, it is possible that gloves were not systematically taken off at that moment. We could not document this phenomenon in our study. Beside shortage of PPE [28] other factors suspected of causing a MDRB epidemic during COVID-19 are work overload of the ICU staff, ICU overcrowding, reinforcement of less experimented staff leading to a decreased in adherence to infection prevention and control measures. $[6,25,26,29]$ Our study was not designed to decipher the respective role of these different mechanisms but we can venture some hypothesis to explain our findings: due to the surge of patients, we had to adapt our usual single room policy creating a new 4-beds ICU with 2 occupants per room. However, staying in our 4-beds ICU was not associated with MDRB acquisition. On the other hand, the number of concerned patients admitted to this ICU was low and no definitive conclusion can be drawn on this point. Regarding prevention and control measures, we were not able to continue our hand hygiene and catheter-dressing audit. An increase in the use of antimicrobials is also a well-known factor associated with MDRB acquisition. [30] Patients with COVID-19, as for other viral infections, [31] were initially suspected to have a high risk of bacterial co-infection and secondary nosocomial infections. [32] In addition, initial COVID-19 symptoms may promote the initiation of antibiotic therapy even when there is no bacterial infection. [25] In line with early descriptions, [5] we observed a large antibiotic use in our study, which was significantly associated with a higher risk of MDRB acquisition in COVID-19 patients even if confounding variables might exist.

Our study has several limitations. First, generalization may be difficult since these results may depend on our center's characteristics. Second, we selected a control group without acute respiratory failure. However, ARDS patients are usually older and have more comorbidities than COVID-19 patients. [33] By contrast, SAH patients offered the advantage of being a cohort of patients with little comorbidity similarly to COVID 19 patients in our center as shown by the Charlson index. Moreover, taking into account predictable differences between the two cohorts, we matched the SAH patients to the COVID-19 patients according to the presence of mechanical ventilation and ICU LOS.

\section{Conclusion}

To conclude, the rate of MDRB acquisition was high in patients admitted in COVID-19. After adjustment, the risk of MDRB acquisition when being admitted to COVID 19 units was higher, but did not reach statistical significance, compared to control patients hospitalized for SAH before the pandemic and 
matched on ICU LOS and the use of mechanical ventilation. Larger multicentric studies will be necessary to assess how the viral pandemic impacts the MDRB one.

\section{Declarations}

Ethics approval and consent to participate: This study was approved by Erasme Hospital Ethics Committee under the protocol number P2020/371. Due to the retrospective nature of the study the need for informed written consent was waived.

Consent for publication: Not applicable

Availability of data and materials: The datasets used and/or analyzed during the current study are available from the corresponding author on reasonable request. All data generated or analyzed during this study are included in this published article [and its supplementary information files].

Competing interests: The authors declare that they have no competing interests

Funding: This research did not receive any specific grant from funding agencies in the public, commercial, or not-for-profit sectors.

Authors' contributions.: DG, FT and EB were responsible for the study concept and design; $\mathrm{EB}, \mathrm{Al}, \mathrm{AG}, \mathrm{SH}$, $D G, F T, J G, A B, N Y, M H, B B, J C$ : acquisition of the data; DG, EB, HN, JLV: analysis and interpretation of the data; DG and EB: drafting of the manuscript; all authors: critical revision of the manuscript for important intellectual content. All authors read and approved the final manuscript.

Acknowledgements: All Erasme teams that worked tirelessly during the epidemic

Authors' information (optional):Not applicable

\section{Abbreviations}

AMR: antimicrobial resistance

CPAP: continuous positive airway pressure

CPE: carbapenemase producing Enterobacteriaceae

ESBL: Extended spectrum beta-lactamase

ICU: Intensive Care Unit

LOS: Length of stay

MDRB: multi drug resistant bacteria 
MRSA: Methicillin resistant Staphylococcus aureus

PPE: Protective personal equipment

SAH: Subarachnoid hemorrhage

SARS-CoV-2 : Severe acute respiratory syndrome coronavirus 2

VRE: Vancomycin resistant Enterococcus

\section{References}

1. Coronavirus disease (COVID-2019) situation reports. World Health Organization. In.; 2020.

2. Li R, Rivers C, Tan Q, Murray MB, Toner E, Lipsitch M: Estimated Demand for US Hospital Inpatient and Intensive Care Unit Beds for Patients With COVID-19 Based on Comparisons With Wuhan and Guangzhou, China. JAMA Netw Open 2020, 3(5):e208297.

3. Garcia Godoy LR, Jones AE, Anderson TN, Fisher CL, Seeley KML, Beeson EA, Zane HK, Peterson JW, Sullivan PD: Facial protection for healthcare workers during pandemics: a scoping review. BMJ Glob Health 2020, 5(5).

4. martin.hirsch@aphp.fr CAGEa: Assistance Publique-Hopitaux de Paris' response to the COVID-19 pandemic. Lancet 2020, 395(10239):1760-1761.

5. Rawson TM, Moore LSP, Zhu N, Ranganathan N, Skolimowska K, Gilchrist M, Satta G, Cooke G, Holmes A: Bacterial and fungal co-infection in individuals with coronavirus: A rapid review to support COVID-19 antimicrobial prescribing. Clin Infect Dis 2020.

6. Rawson TM, Moore LSP, Castro-Sanchez E, Charani E, Davies F, Satta G, Ellington MJ, Holmes AH: COVID-19 and the potential long-term impact on antimicrobial resistance. J Antimicrob Chemother 2020.

7. Huttner A, Harbarth S, Carlet J, Cosgrove S, Goossens H, Holmes A, Jarlier V, Voss A, Pittet D: Antimicrobial resistance: a global view from the 2013 World Healthcare-Associated Infections Forum. Antimicrob Resist Infect Control 2013, 2:31.

8. Gutierrez-Gutierrez B, Rodriguez-Bano J: Current options for the treatment of infections due to extended-spectrum beta-lactamase-producing Enterobacteriaceae in different groups of patients. Clin Microbiol Infect 2019, 25(8):932-942.

9. Falagas ME, Tansarli GS, Karageorgopoulos DE, Vardakas KZ: Deaths attributable to carbapenemresistant Enterobacteriaceae infections. Emerg Infect Dis 2014, 20(7):1170-1175.

10. Antibiotic resistance threats in the United States, 2013. . In: CDC. 2013.

11. Brusselaers $N$, Vogelaers $D$, Blot $S$ : The rising problem of antimicrobial resistance in the intensive care unit. Ann Intensive Care 2011, 1:47.

12. Schenck EJ, Hoffman K, Goyal P, Choi J, Torres L, Rajwani K, Tam CW, Ivascu N, Martinez FJ, Berlin DA: Respiratory Mechanics and Gas Exchange in COVID-19 Associated Respiratory Failure. Ann Am 
Thorac Soc 2020.

13. Taccone FS, Badenes R, Arib S, Rubulotta F, Mirek S, Franchi F, Gordon S, Nadji A, Crippa IA, Stazi E et al: Cerebrospinal Fluid Glucose and Lactate Levels After Subarachnoid Hemorrhage: A Multicenter Retrospective Study. J Neurosurg Anesthesiol 2020, 32(2):170-176.

14. Witiw CD, Ibrahim GM, Fallah A, Macdonald RL: Early predictors of prolonged stay in a critical care unit following aneurysmal subarachnoid hemorrhage. Neurocrit Care 2013, 18(3):291-297.

15. Alaraj A, Hussein AE, Esfahani DR, Amin-Hanjani S, Aletich VA, Charbel FT: Reducing length of stay in aneurysmal subarachnoid hemorrhage: A three year institutional experience. J Clin Neurosci 2017, 42:66-70.

16. The European Committee on Antimicrobial Susceptibility Testing. Breakpoint tables for interpretation of MICs and zone diameters. In: Version 90, 2019.

17. Magiorakos AP, Srinivasan A, Carey RB, Carmeli Y, Falagas ME, Giske CG, Harbarth S, Hindler JF, Kahlmeter G, Olsson-Liljequist B et al: Multidrug-resistant, extensively drug-resistant and pandrugresistant bacteria: an international expert proposal for interim standard definitions for acquired resistance. Clin Microbiol Infect 2012, 18(3):268-281.

18. De Waele JJ, Boelens J, Leroux-Roels I: Multidrug-resistant bacteria in ICU: fact or myth. Curr Opin Anaesthesiol 2020, 33(2):156-161.

19. De Bus L, Denys W, Catteeuw J, Gadeyne B, Vermeulen K, Boelens J, Claeys G, De Waele JJ, Decruyenaere J, Depuydt PO: Impact of de-escalation of beta-lactam antibiotics on the emergence of antibiotic resistance in ICU patients: a retrospective observational study. Intensive Care Med 2016, 42(6):1029-1039.

20. Kerneis S, Lucet JC: Controlling the Diffusion of Multidrug-Resistant Organisms in Intensive Care Units. Semin Respir Crit Care Med 2019, 40(4):558-568.

21. Grundmann H, Hahn A, Ehrenstein B, Geiger K, Just H, Daschner FD: Detection of cross-transmission of multiresistant Gram-negative bacilli and Staphylococcus aureus in adult intensive care units by routine typing of clinical isolates. Clin Microbiol Infect 1999, 5(7):355-363.

22. Chetchotisakd P, Phelps CL, Hartstein Al: Assessment of bacterial cross-transmission as a cause of infections in patients in intensive care units. Clin Infect Dis 1994, 18(6):929-937.

23. Boyce JM, Pittet D, Healthcare Infection Control Practices Advisory C, Force HSAIHHT: Guideline for Hand Hygiene in Health-Care Settings. Recommendations of the Healthcare Infection Control Practices Advisory Committee and the HIPAC/SHEA/APIC/IDSA Hand Hygiene Task Force. Am J Infect Control 2002, 30(8):S1-46.

24. Osterholm MT: Preparing for the next pandemic. N Engl J Med 2005, 352(18):1839-1842.

25. Nieuwlaat R, Mbuagbaw L, Mertz D, Burrows L, Bowdish DME, Moja L, Wright GD, Schunemann HJ: COVID-19 and Antimicrobial Resistance: Parallel and Interacting Health Emergencies. Clin Infect Dis 2020.

26. Clancy CJ, Nguyen MH: COVID-19, superinfections and antimicrobial development: What can we expect? Clin Infect Dis 2020. 
27. Sturdy A, Basarab M, Cotter M, Hager K, Shakespeare D, Shah N, Randall P, Spray D, Arnold A: Severe COVID-19 and Healthcare Associated Infections on the ICU: Time to Remember the Basics? J Hosp Infect 2020.

28. Ranney ML, Griffeth V, Jha AK: Critical Supply Shortages - The Need for Ventilators and Personal Protective Equipment during the Covid-19 Pandemic. N Engl J Med 2020, 382(18):e41.

29. Murray AK: The Novel Coronavirus COVID-19 Outbreak: Global Implications for Antimicrobial Resistance. Front Microbiol 2020, 11:1020.

30. Chatzopoulou M, Reynolds L: Role of antimicrobial restrictions in bacterial resistance control: a systematic literature review. J Hosp Infect 2020, 104(2):125-136.

31. Morens DM, Taubenberger JK, Fauci AS: Predominant role of bacterial pneumonia as a cause of death in pandemic influenza: implications for pandemic influenza preparedness. $J$ Infect Dis 2008, 198(7):962-970.

32. Zhou F, Yu T, Du R, Fan G, Liu Y, Liu Z, Xiang J, Wang Y, Song B, Gu X et al: Clinical course and risk factors for mortality of adult inpatients with COVID-19 in Wuhan, China: a retrospective cohort study. Lancet 2020, 395(10229):1054-1062.

33. Burn E, You SC, Sena AG, Kostka K, Abedtash H, Abrahao MTF, Alberga A, Alghoul H, Alser O, Alshammari TM et al: An international characterisation of patients hospitalised with COVID-19 and a comparison with those previously hospitalised with influenza. medRxiv 2020.

\section{Tables}

Table 1: Characteristics of patients that did (MDRB+) or didn't (MDRB-) acquire MDRB during their COVID 19 ICU stay 


\begin{tabular}{|c|c|c|c|c|}
\hline & $\begin{array}{l}\text { All } \\
\text { patients } \\
(\mathrm{N}=72)\end{array}$ & $\begin{array}{l}\text { MDRB - } \\
(\mathrm{N}=48)\end{array}$ & $\begin{array}{l}\text { MDRB + } \\
(\mathrm{N}=24)\end{array}$ & $\begin{array}{l}p^{* *} \\
\text { value }\end{array}$ \\
\hline Age; years, mean ( $\pm S D)$ & $61( \pm 14)$ & $62( \pm 15)$ & $61( \pm 9)$ & 0.84 \\
\hline Male gender, n (\%) & $47(65)$ & $30(63)$ & $17(71)$ & 0.60 \\
\hline Charlson comorbidity index, median (IQR) & $1(0-3)$ & $1(0-3)$ & $1(0-4)$ & 0.77 \\
\hline Hospitalization in the last 6 months, $\mathrm{n}(\%)$ & $11(15)$ & $9(19)$ & $2(8)$ & 0.32 \\
\hline Patients MDRB + at admission, $\mathrm{n}(\%)$ & $5(7)$ & $4(8)$ & $1(4)$ & 0.66 \\
\hline SAPS 3 score, median (IQR) & $56(47-69)$ & $\begin{array}{l}56(47- \\
70)\end{array}$ & $\begin{array}{l}57(47- \\
65)\end{array}$ & 0.95 \\
\hline SOFA sore, median (IQR) & $6(3-9)$ & $6(3-10)$ & $7(5-9)$ & 0.39 \\
\hline Central venous catheter*, n (\%) & $68(94)$ & $45(93)$ & $23(96)$ & 0.99 \\
\hline Urinary tract catheter*, n (\%) & $65(90)$ & $43(90)$ & $22(92)$ & 0.99 \\
\hline Mechanical ventilation*, n (\%) & $40(56)$ & $24(50)$ & $16(67)$ & 0.22 \\
\hline Length of MV*; days, median (IQR) & $3(0-12)$ & $1(0-11)$ & $7(0-15)$ & 0.27 \\
\hline Vasopressor use, n (\%) & $50(69)$ & $28(58)$ & $22(92)$ & 0.006 \\
\hline Renal replacement therapy, n (\%) & $17(24)$ & $10(21)$ & $7(29)$ & 0.56 \\
\hline ECMO, n (\%) & $11(15)$ & $7(15)$ & $4(17)$ & 0.99 \\
\hline Surgery*, n (\%) & $10(14)$ & $8(17)$ & $2(8)$ & 0.48 \\
\hline Antimicrobial therapy *, n (\%) & $56(78)$ & $34(71)$ & $22(92)$ & 0.05 \\
\hline $\begin{array}{l}\text { Length of Antimicrobial therapy*; days, median } \\
\text { (IQR) }\end{array}$ & $5(2-7)$ & $4(0-7)$ & $6(4-7)$ & 0.09 \\
\hline Length of exposure*; days, median (IQR) & $9(4-18)$ & $5(2-18)$ & $12(8-18)$ & 0.02 \\
\hline Admission to double bed ICU room*, n (\%) & $8(11)$ & $4(8)$ & $4(17)$ & 0.43 \\
\hline ICU LOS; days, median (IQR) & $11(3-28)$ & $6(2-18)$ & $\begin{array}{l}28(17- \\
32)\end{array}$ & $<0.001$ \\
\hline ICU LOS of survivors; days, median (IQR) & $7(2-28)$ & $4(2-9)$ & $\begin{array}{l}26(24- \\
28)\end{array}$ & 0.001 \\
\hline Hospital LOS; days, median (IQR) & $24(12-45)$ & $\begin{array}{l}19(11- \\
34)\end{array}$ & $\begin{array}{l}39(21- \\
61)\end{array}$ & 0.005 \\
\hline
\end{tabular}




\begin{tabular}{|lllll|}
\hline Hospital LOS of survivors; days, median (IQR) & $33(16-52)$ & $20(11-$ & $53(33-$ & 0.002 \\
\hline ICU mortality, n(\%) & & $39)$ & $69)$ & \\
\hline Hospital mortality, n(\%) & $22(31)$ & $16(33)$ & $6(25)$ & 0.59 \\
\hline
\end{tabular}

*variables collected from admission until MDRB acquisition.

**p value was calculated using Qui square test, Fisher exact test, t-Student test or Mann-Whitney as appropriate.

MV: mechanical ventilation; SAPS 3: Simplified Acute Physiology Score III; SOFA: Sequential organ failure assessment; ICU: Intensive Care Unit; ECMO: Extracorporeal membrane oxygenation; IQR: Interquartile range; LOS: length of stay;

Table 2: Comparison between patients admitted to COVID 19 ICUs and subarachnoid hemorrhage patients admitted to medical-surgical ICUs 


\begin{tabular}{|c|c|c|c|}
\hline & & & p-value ${ }^{\#}$ \\
\hline & $N=72$ & $N=72$ & \\
\hline Age; years, mean $( \pm S D)$ & $53( \pm 16)$ & $61( \pm 14)$ & $<0.001$ \\
\hline Male gender, $\mathrm{n}(\%)$ & $32(44)$ & $47(65)$ & 0.02 \\
\hline Charlson comorbidity index, median (IQR) & $1(0-3)$ & $1(0-3)$ & 0.68 \\
\hline Hospitalization in the previous 6 months, $\mathrm{n}(\%)$ & $3(4)$ & $11(15)$ & 0.02 \\
\hline Transfer from another hospital $\star \star ~$ & $0(0)$ & $20(28)$ & $<0.001$ \\
\hline MDRB + at admission, $\mathrm{n}(\%)^{\star \star \star}$ & $4(6)$ & $5(7)$ & 0.99 \\
\hline SAPS 3 score, median (IQR) & $33(27-39)$ & $56(47-69)$ & $<0.001$ \\
\hline SOFA sore, median (IQR) & $5(1-10)$ & $6(3-9)$ & 0.08 \\
\hline Central venous catheter*, n (\%) & $44(61)$ & $68(94)$ & $<0.001$ \\
\hline Urinary tract catheter*, n (\%) & $41(57)$ & $65(90)$ & $<0.001$ \\
\hline Mechanical ventilation, $\mathrm{n}(\%)$ & $52(72)$ & $52(72)$ & 1.0 \\
\hline Length of $\mathrm{MV} *$; days, median (IQR) & $1(0-10)$ & $3(0-12)$ & 0.55 \\
\hline Vasopressor, n (\%) & $44(61)$ & $50(69)$ & 0.38 \\
\hline Renal replacement therapy, n (\%) & $1(1)$ & $17(24)$ & $<0.001$ \\
\hline Antimicrobial therapy*, n (\%) & $37(51)$ & $56(78)$ & 0.002 \\
\hline Length of Antimicrobial therapy * days, median (IQR) & $1(0-5)$ & $5(2-7)$ & $<0.001$ \\
\hline Length of exposure; days, median (IQR) & $10(4-22)$ & $9(4-18)$ & 0.67 \\
\hline MDRB acquisition during ICU stay, n (\%) & $16(22)$ & $24(33)$ & 0.19 \\
\hline ICU LOS; days, median (IQR) & $11(3-26)$ & $11(3-28)$ & 0.86 \\
\hline ICU LOS of survivors; days, median (IQR) & $10(3-27)$ & $7(2-28)$ & 0.98 \\
\hline ICU mortality, n (\%) & $16(22)$ & $22(31)$ & 0.26 \\
\hline
\end{tabular}

*variables collected from admission until MDRB acquisition.

** defined as hospital stay $>48 \mathrm{~h}$ before ICU admission in our center.

$\star \star \star$ index cases 
\# p-values were calculated using Qui square or Fisher test, T-student or Mann-Whitney as appropriate.

SAPS 3: Simplified Acute Physiology Score III; SOFA: Sequential organ failure assessment; ICU: Intensive Care Unit; ECMO: Extracorporeal membrane oxygenation; IQR: Interquartile range; MDRB: multidrug resistant bacteria; MV: mechanical ventilation

Table 3: Fine and Gray competing risk analysis for factors related to MDRB acquisition

\begin{tabular}{|lll|}
\hline & $\begin{array}{l}\text { Univariable } \\
\text { sHR (Cl 95\%) }\end{array}$ & $\begin{array}{l}\text { Multivariable } \\
\text { sHR (Cl 95\%) }\end{array}$ \\
\hline SOFA score & $0.97(0.89-1.05)$ & \\
\hline Mechanical ventilation & $0.58(0.30-1.11)$ & $0.61(0.3-1.26)$ \\
\hline Central venous catheter & $0.54(0.26-1.13)$ & $0.66(0.27-1.62)$ \\
\hline Urinary tract catheter & $0.98(0.41-2.33)$ & \\
\hline Antimicrobial therapy & $1.32(0.48-3.61)$ & \\
\hline Admission to COVID-19 ICUs & $1.62(0.88-2.99)$ & $1.71(0.93-3.12)$ \\
\hline
\end{tabular}

All variables were considered before MDRB acquisition. COVID-19 ICUs: coronavirus disease 19 intensive care units; SHR: sub-distribution hazard ratio. These results were obtained by the Fine and Gray method for competing risk analysis.

\section{Figures}

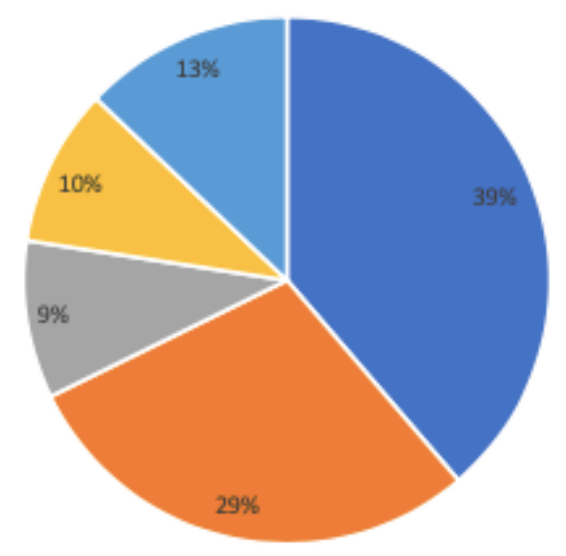

- AmpC Enterobacteriaceae

- Extended Spectrum BetaLactamase Enterobacteriaceae

- Carbapenem Resistant Enterobacteriaceae

- Vancamycin Resistant Enterococo

- MDR Pseudamanas aeruginasa

Figure 1 
Title: Distribution of MDRB bacteria acquired during COVID 19- ICU stay according to mechanism of resistance Legend: AmpC $\beta$ lactamase (AmpC) Enterobacteriaceae $(N=12)$ : Escherichia coli $(N=1)$; Enterobacter aerogenes $(\mathrm{N}=6)$; Enterobacter cloacae $(\mathrm{N}=5)$ Extended spectrum $\beta$-lactamase $(\mathrm{ESBL})$ Enterobacteriaceae $(N=9)$ : Enterobacter aerogenes $(N=1)$, Escherichia coli $(N=2)$, Klebsiella pneumoniae $(\mathrm{N}=6)$ Carbapenem resistant Enterobacteriaceae (CRE) $\mathrm{N}=3$ : Enterobacter aerogenes $(\mathrm{N}=1)$, Klebsiella pneumoniae ( $\mathrm{N}=2$ ) Vancomycin resistant enterococci (VRE) $\mathrm{N}=3$ : Enterococcus faecium ( $\mathrm{N}=3$ ) Multi-drug resistant Pseudomonas aeruginosa $(\mathrm{N}=4)$

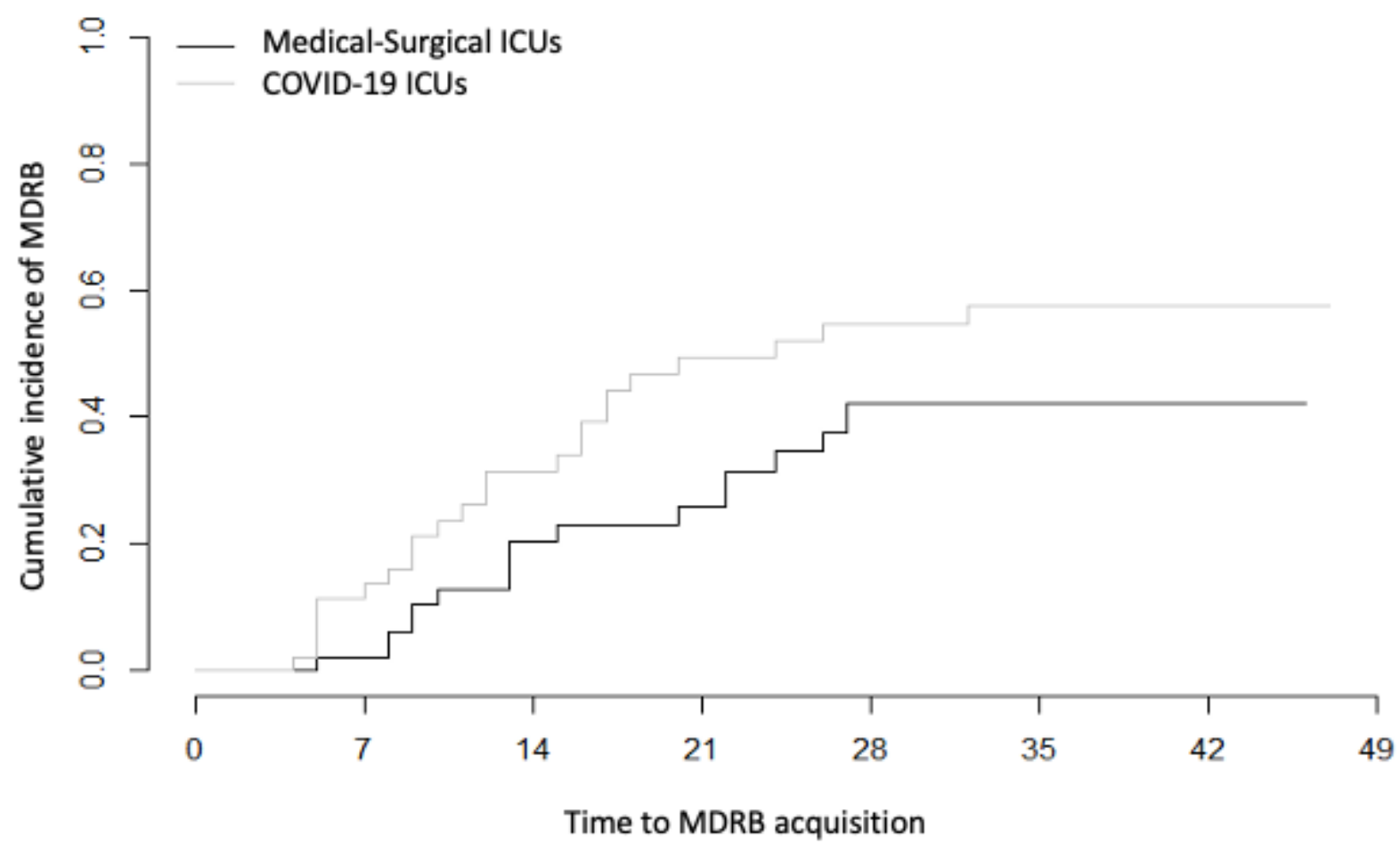

Figure 2

Title: Cumulative incidence of MDRB over time during ICU admission into two types of units (Medicalsurgical and COVID 19 units). Legend: Statistical significance was calculated using the Fine and Gray method. $p=0.14$

\section{Supplementary Files}

This is a list of supplementary files associated with this preprint. Click to download.

- SupplemementalelectronicmaterialARIC.docx 